Canadian University Music Review

Canadian University Music Review

Revue de musique des universités canadiennes

\title{
Glenn Gould, entretiens avec Jonathan Cott, traduit et présenté par Jacques Drillon. Paris : Lattès, Collection Musiques et Musiciens, 1983, 156 pp.
}

\section{Ghyslaine Guertin-Bélanger}

\section{Numéro 5, 1984}

URI : https://id.erudit.org/iderudit/1014020ar

DOI : https://doi.org/10.7202/1014020ar

Aller au sommaire du numéro

\section{Éditeur(s)}

Canadian University Music Society / Société de musique des universités canadiennes

\section{ISSN}

0710-0353 (imprimé)

2291-2436 (numérique)

Découvrir la revue

Citer ce compte rendu

Guertin-Bélanger, G. (1984). Compte rendu de [Glenn Gould, entretiens avec Jonathan Cott, traduit et présenté par Jacques Drillon. Paris : Lattès, Collection Musiques et Musiciens, 1983, 156 pp.] Canadian University Music Review / Revue de musique des universités canadiennes, (5), 357-360.

https://doi.org/10.7202/1014020ar

All Rights Reserved (C Canadian University Music Society / Société de musique des universités canadiennes, 1984
Ce document est protégé par la loi sur le droit d'auteur. L'utilisation des services d'Érudit (y compris la reproduction) est assujettie à sa politique d'utilisation que vous pouvez consulter en ligne.

https://apropos.erudit.org/fr/usagers/politique-dutilisation/ 
et « sa connaissance pratique de la musique » qu'il a su combiner pour comprendre la pensée musicale de Gould.

Les biographes ne possèdent pas tous cette qualité d'objectivité que Gould attribue à Payzant. Dans son essai critique, Boulez-le-terrible, Gould dénonce l'ouvrage de Joan Peyser qui décrit le portrait "psychobiographique " du compositeur Pierre Boulez, en attirant l'attention du lecteur sur les expériences tumultueuses de sa vie, tissée de "complots, de contre-complots, de brouilles, de réconciliations ». Peyser aurait été, selon Gould, "la proie des fantasmes de la mode ». Son ouvrage n'est rien d'autres qu'une pièce dont la moralité serait : " celui qui vit par le glaive périra par le glaive ». Mais, de continuer Gould, on ne saurait douter que «la sympathie de Peyser va au glaive plutôt qu'à sa victime " (p. 282).

Entre ces deux essais critiques, Gould exprime son admiration inébranlable pour Barbara Streisand en procédant à la recension d'un disque, "Classical Barbara », paru en 1976 pour la maison Columbia. Il établit une comparaison entre l'art de cette interprète et celui d'Elizabeth Schwarzkopf. Ses affirmations dithyrambiques à l'égard de Streisand reposent sur une bonne connaissance de la technique vocale de l'interprète et des particularités de son style d'expression. Il emprunte à sa sensibilité et à son sens analytique, les instruments nécessaires pour démontrer que le disque "Classical Barbara " n'est pas vraiment bon ni vraiment mauvais (p. 274).

Si Glenn Gould est "le dernier puritain » (ses écrits nous ont permis de le constater), il est aussi paradoxalement " un homme du futur ». Bien avant qu'il soit question du "virage technologique ", Gould a su comprendre et valoriser la nature et le rôle de la technologie dans le domaine de l'art musical. Sa réflexion est non seulement au centre même des préoccupations socio-culturelles de notre époque, mais elle rejoint par son questionnement une dimension laissée pour compte par les tenants de la modernité. L'ouvre littéraire de Gould constitue un instrument essentiel à la reconstitution de sa " poïétique ", c'est-à-dire de ses intentions esthétiques, philosophiques, des conditions matérielles, psychologiques susceptibles de nous faire comprendre les choix qui l'ont guidé dans son interprétation créatrice. Plus qu'un instrument de travail, les écrits de Gould, par la beauté et l'originalité de l'écriture, invitent le lecteur à découvrir le plaisir du texte.

Ghyslaine Guertin-Bélanger

Glenn Gould, entretiens avec Jonathan Cott, traduit et présenté par Jacques Drillon. Paris : Lattès, Collection Musiques et Musiciens, 1983, $156 \mathrm{pp}$.

Les entretiens avec le pianiste canadien Glenn Gould réalisés par 
le journaliste américain Jonathan Cott ont déjà fait l'objet d'une publication dans le magazine Rolling Stone, en août 1974. La présentation et la traduction de ces entretiens par Jacques Drillon constituent par ailleurs le premier livre à paraître en français sur Gould. Cette publication s'ajoute à la bibliographie sur Gould qui compte déjà en anglais les deux ouvrages suivants : Geoffrey Payzant, Glenn Gould : Music and Mind (Toronto: Van Nostrand Reinhold, 1978) - qui vient d'être traduit en français aux éditions Fayard (1984) - et John McGreevy, éd., Glenn Gould : Variations (Toronto : Doubleday, 1983).

Le livre se compose d'une préface de quarante pages, intitulée Le Plaisir et l'extase, signée par le traducteur. Les entretiens entre Gould et Cott qui font suite à cette préface se divisent en deux parties et couvrent une centaine de pages. Le tout se termine sur une courte biographie de Gould et une discographie non commentée.

La préface de Jacques Drillon, dans laquelle l'explication de l'esthétique de Gould devient l'occasion d'un étalage de références littéraires, déçoit par ses résonances faussement profondes. D'emblée, l'intérêt du livre réside dans les entretiens entre Gould et Cott. Gould nous parle de ses expériences d'enregistrement, de son besoin de solitude, de Mozart et de sujets tout aussi étonnants que les Beatles et Petula Clark. À travers ces propos diversifiés qui suivent un cours décousu, Gould nous livre sa "manière de faire ". Il nous révèle les grandes lignes de son esthétique créatrice fondée sur une approche à la fois analytique et expérimentale de la musique.

Pour Gould, l'ouvre d'art musicale est avant tout structure. Elle est constituée d'un ensemble de formes. Il revient à l'interprète de les créer, de les révéler et, partant, d'exprimer la signification intrinsèque du discours musical. L'interprétation musicale demeure pour Gould une activité de l'esprit. Son idéal de " perfection analytique " qu'il décrit dans la première partie des entretiens avec Cott, contribue à l'illustrer.

De même, les termes avec lesquels Gould exprime son admiration pour le pianiste Arthur Schnabel, reflètent sa théorie esthétique. Gould reconnaît en ce musicien le meilleur interprète de Beethoven parce qu'il aurait réussi à faire connaître clairement "le sens de la structure " dans la plupart de ses ouvres. C'est encore à cette dimension apollinienne de la musique que Gould se réfère lorsqu'il se tourne du côté des compositeurs qu'il affectionne. Bach détient la première place en tant que meilleur contrapuntiste. Orlando Gibbons demeure aussi son compositeur préféré. Il aime le premier Mozart, il s'avoue un mordu de Schoenberg et un "wagnérien absolu, malade incurable de toutes ses ouvres de la fin» (p. 91).

En quoi consiste ce rapport de Gould à Wagner qui, au départ, peut paraître contradictoire? Gould fournit au lecteur les éléments nécessaires pour en comprendre les principales articulations lorsqu'il expose les principes qui l'ont guidé dans sa réalisation des transcrip- 
tions pour le piano de Siegfried Idyll, le prélude des Maîtres Chanteurs et l'Aube et le Voyage de Siegfried sur le Rhin. Ces trois oeuvres intéressent Gould par leur organisation contrapuntique et leur thématique. Après avoir expliqué sa conception de la transcription qui repose entièrement sur le refus d'accepter la version orchestrale comme modèle platonicien, Gould décrit son savoir-faire. Sa démarche en tant que traducteur rejoint celle de l'interprète. Elle comporte une activité de sélection, un parti pris. Gould projette sur l'ouvre un « éclairage " nouveau en ne s'intéressant qu'à la jubilation de la forme. Commettrait-il alors un contresens vis-à-vis de Wagner? Serait-il infidèle au compositeur?

C'est le reproche que lui aurait adressé la critique musicale à propos, entre autres, de son interprétation des sonates et du concerto en do mineur de Mozart. Gould, toujours dans la première partie des entretiens avec Cott, répond à ses détracteurs qui l'auraient accusé d'avoir ajouté dans le concerto, des " ornementations " et des " passages de continuo ". Il se tourne du côté de Mozart en affirmant que ce compositeur « en faisant autant. Et, qui plus est, il trouvait normal que les autres le fassent " (p. 73). En ce qui concerne l'interprétation des sonates, Cott lui reproche ses " tempi furieux, dépressifs » ... alors que d'autres critiques ont attaqué la carrure de son interprétation antimozartienne. Gould affirme avoir joué cette musique " dans une sorte de continuité baroque" (p. 79). Son objectif consistait encore cette fois à exprimer la structure de l'œuvre "sans tenir compte d'un tempo précis " (p. 79). Le reste de ses propos sur Mozart sont provocateurs à souhait. Mozart n'aurait pas, selon Gould, l'intelligence du développement par exemple.

Dans la seconde partie des entretiens avec Cott, Gould rend compte de ses diverses expériences d'enregistrement. L'appareillage technique est au service de l'analyse et de la dissection de l'œuvre. La description de sa réalisation en tétraphonie d'une fugue de Bach et plus particulièrement de la Sonate $n^{\circ} 5$ de Scriabine l'illustre bien. Gould croit néanmoins que l'avenir de la tétraphonie se situe davantage au niveau de la parole que de la musique.

À la demande de Cott, Gould aborde par la suite la question de ses documentaires radiophoniques axés sur le thème de la solitude et de l'isolement. Gould retient entre autres celui qu'il a réalisé sur Stokowski pour "le moment magique " qu'il contient. C'est avec enthousiasme qu'il explique comment il est parvenu à illustrer l'histoire de l'expérience des enregistrements de ce musicien et à suggérer, par le biais de l'enregistrement, une de ses phrases "élégiaques": "Aujourd'hui, c'est mieux qu'hier, mais nous devrions pouvoir faire mieux encore " (p. 116). L'objectif que poursuit Gould dans cette expérience, tout comme d'ailleurs dans ses deux autres réalisations : The Idea of North, The Latecomers, consiste à vouloir comprendre le comportement soit d'un individu, soit d'un groupe d'individus qui ont 
choisi de s'isoler. Cette recherche que Gould effectue autour du problème existentiel de la solitude rejoint un de ses fantasmes : celui " de passer au moins un hiver entier au nord du cercle arctique (...) au moment où le soleil est couché » (p. 125) afin d'y retrouver la qualité de solitude propre "à rétablir l'équilibre ".

Gould nous rappelle, en terminant, ses principales exigences créatrices. La critique qu'il adresse aux Beatles permet de les retracer. Il leur reproche l'indigence de la structure harmonique de leurs compositions et leur préfère Petula Clark ou Barbara Streisand à laquelle il attribue une "pensée structurée " et un grand "sens de l'unité ". Gould rend plus explicite l'essentiel de son message lorsqu'il déclare :

On prétend que l'invention doit faire du bruit (...) Au lieu de bruit, je vois plutôt de la subtilité, celle avec laquelle on pose des prémisses différentes de celles qu'on attendait de vous (p. 137).

Gould nous a démontré tout au long de ces entretiens avec Jonathan Cott en quoi consistait cette aventure. Il nous a fait connaître le risque aussi qu'elle comportait. Il revient au lecteur-auditeur de la découvrir, de la comprendre et de l'interpréter à son tour au moyen de sa propre expérience musicale.

Ghyslaine Guertin-Bélanger

Les fantaisies du voyageur (XXXIII Variations Schaeffner). Paris: Société française de musicologie, 1982, 408 pp.

André Schaeffner (1895-1980) est à coup sûr l'une des personnalités les plus importantes de la musicologie française. Son ouvrage fondamental, Origine des instruments de musique (1936), a fait de lui un des "grands " de l'ethnomusicologie, discipline dont il a proposé l'appellation moderne en remplacement de "musicologie comparée " (vergleichende Musikwissenschaft) non sans quelques regrets (Variations, p. 376 et Schaeffner 1980: 10). Auteur d'un des classiques de l'organologie, chercheur situé au confluent de l'ethnologie et de la musicologie, il se définissait lui-même comme un historien et un sociologue de la musique (Schaeffner 1980:10).

C'est donc la dimension et la diversité des intérêts et des travaux d'André Schaeffner que permet tout d'abord de mesurer le volume d'hommage préparé par $\mathrm{M}^{\mathrm{me}}$ Denise Paulme-Schaeffner, sa veuve, avec le concours de Jean Gergely et de Gilbert Rouget. La bibliographie établie par Jean-Michel Nectoux (pp. 401-408), la note biographique de $\mathrm{M}^{\mathrm{me}}$ Schaeffner (pp. 363-365), la transcription d'une conversation menée par Charles Duvelle (pp. 366-380), la situation de l'ethnomusicologue et de musicologue par Gilbert Rouget et François Lesure (pp. 3-15) et divers témoignages personnels (Marcel Belvianes, Iannis 